ISSN 1112-9867

\title{
MAPPING OF LAND-COVER FROM REMOTELY SENSED IMAGES IN THE VALLEY OF OUED RIGH (ALGERIAN SOUTHEAST)
}

\author{
N. Boussaada-Maabdi ${ }^{1,2^{*}}$ H. Bousnoubra-Kherici ${ }^{1}$, N. $\operatorname{Kherici}^{1}$, N. $\operatorname{Hammad}^{2}$ \\ ${ }^{1}$ Université Badji Moukhtar, BP N ${ }^{\circ} 12$ El Hadjar 23200 Annaba, Algérie. \\ ${ }^{2}$ Université Kasdi Merbah, Laboratoire de Géologie du Sahara, BP 511, Route Ghardaïa, \\ 30000 Ouargla, Algérie.
}

Received: 16 October 2016 / Accepted: 18 April 2017 / Published online: 01 May 2017

\begin{abstract}
The aim of this work is to analyze the evolution of arid areas by remote sensing and to assess the extent of landscape changes over time. The study consists of a multitemporal analysis of LANDSAT TM and ETM + satellite images from 1987, 2000 and 2009 covering the Oued Righ valley in south-eastern Algeria.

Numerous colored compositions carried out, allowed us a very good visual characterization of the surface states. Supervised classification charts, carried at the second step, are of good quality as indicated by the precision indices and Kappa indices from the various confusion matrices.

The change maps obtained by diachronic analysis of the classifications carried out show a clear modification of the natural landscape with a significant increase in the surface of the water, the number of the population and a reduction of the palm groves.
\end{abstract}

Keywords: Remote sensing; Change mapping; land cover; multitemporal analysis; Northern Sahara.

Author Correspondence, e-mail: houria.kherici@univ-annaba.org doi: http://dx.doi.org/10.4314/jfas.v9i2.6 


\section{INTRODUCTION}

L'augmentation de la demande en eau, place la bonne gestion de cette ressource au centre des préoccupations mondiales, et lui confère une attention particulière. Depuis longtemps, dirigeants et chercheurs [1] concourent à trouver un moyen adéquat de gestion des eaux notamment dans les zones arides où le renouvellement et l'alimentation des nappes deviennent de plus en plus rarissimes.

Le traitement classique des données devient limité et très lourd à manipuler, d'où la nécessité d'une nouvelle technologie d'acquisition et de traitement de l'information plus adéquate pour une vision globale. Cette dernière montre son apport dans les aspects de cartographie, de prospection, de suivi qualitatif et de gestion et constitue un moyen pour remédier aux problèmes de l'hydrogéologue et/ou du gestionnaire [2].

De même que pour l'ensemble des options des sciences de la Terre, La télédétection, fait preuve de sa capacité pour l'étude hydrologique et hydrogéologique. De nombreux auteurs [311] ont étudiés l'évolution des états des sols (salinité, couvert végétal ....) par imagerie satellitaire aussi bien en superficie restreinte qu'en vastes étendues.

C'est dans cette optique que s'inscrit cette étude consacrée au suivi de l'évolution des ressources hydriques dans une partie du Sahara Septentrional algérien où l'utilisation de la télédétection devient désormais incontournable. Cette technique admet d'analyser le milieu d'une manière exhaustive et surtout plus dynamique sur de grandes surfaces pour arriver à une meilleure approche de l'espace au lieu d'une mesure ponctuelle.

Cette étude, à caractère essentiellement cartographique multi-dates, tente de répondre à de multiples interrogations telles: Observe-t-on des phénomènes de progression des remontées spectaculaires (chotts), augmentation du nombre des palmeraies, salinité des sols? Quelle sont les structures spatiale des changements à l'échelle régionale ? Peut-on constater d'autres évolutions durant les années à venir ?

Pour atteindre cet objectif, nous avons utilisé trois scènes LANDSAT (TM et ETM+) à dates différentes 1987,2000 et 2009, couvrant la zone d'étude.

En effet, cette approche comparative permet facilement de détecter les modifications liées au changement climatique.

\subsection{Description de la zone d'étude}

La zone d'étude « la vallée de l'Oued Righ » appartenant au Bas Sahara (à 600 km au Sud de la capital, Alger), couvre une superficie de 3750 ha. Allongée sur environ $150 \mathrm{~km}$ sur un axe Nord Sud, cette vallée est limitée au Nord par le chott Merouane et au Sud par El Goug (fig. 1). Sa topographie est très plane, s'approchant de $1 \%$. Son étendue couvre plus d'une 
cinquantaine d'oasis où la culture des dattiers reste la seule et unique ressource socioéconomique.

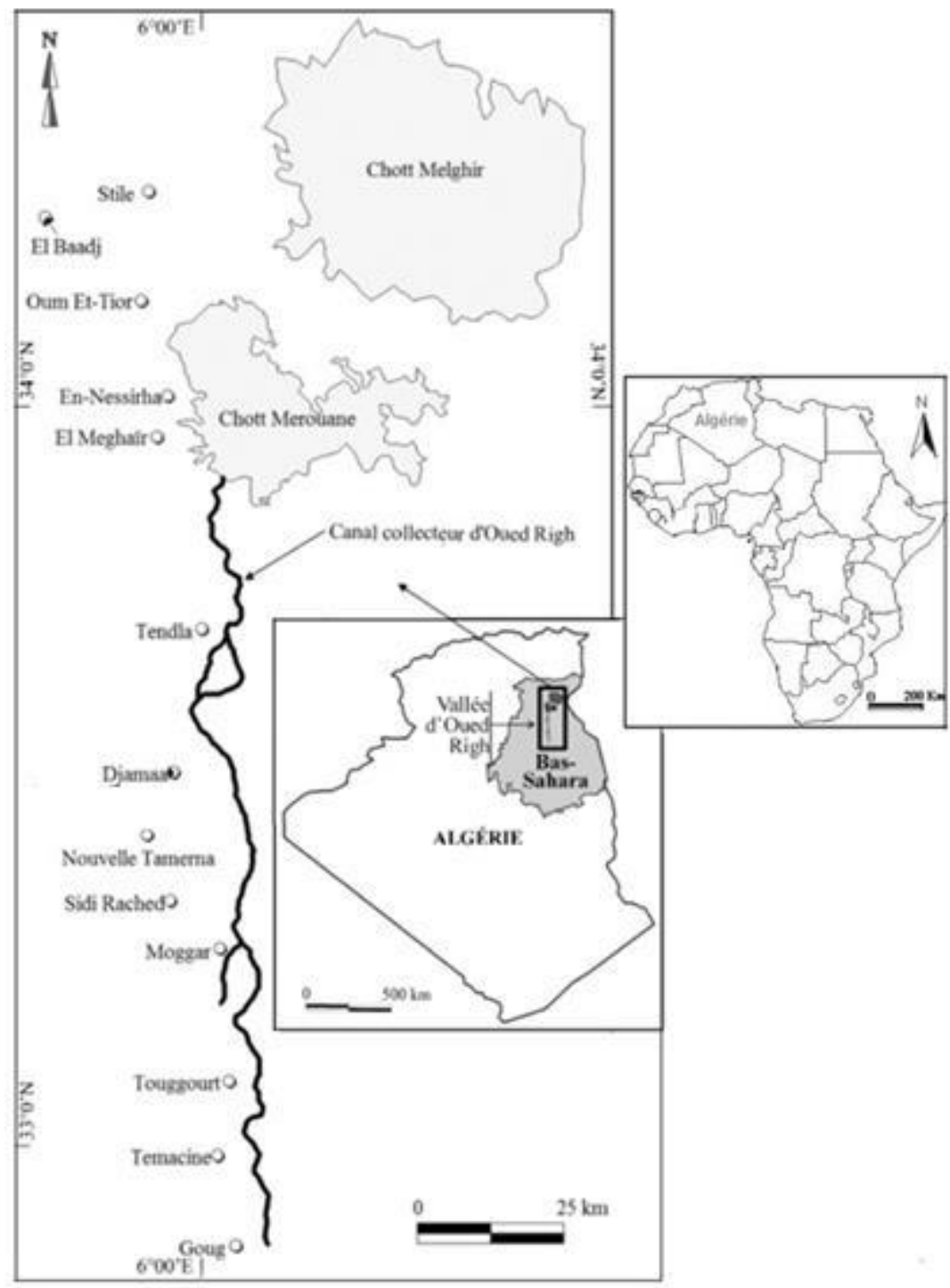

Fig.1. Situation de la vallée d'Oued Righ.[12]

Les sols de la région ont une texture fine limono-argileuse, avec des teneurs élevées en sels au Nord (au-delà d'El Meghair) et grossière à dominance sableuse au Sud, tandis que le fond est comblé par des sédiments sableux entrecoupés de lentilles d'argile salifère.

\subsection{Contexte climatique et hydrologique}

Le climat est désertique ou hyper aride caractérisé par des précipitations faibles à rares et irrégulières ne dépassant pas les $70 \mathrm{~mm} / \mathrm{an}$, suite à des orages violents qui génèrent des ruissellements. Ces dernières sont vite reprises par la forte évaporation résultant des températures élevées $\left(22^{\circ} \mathrm{C}\right.$ en moyenne annuelle et $34^{\circ} \mathrm{C}$ au mois le plus chaud)et ne jouent 
pratiquement aucun rôle dans la recharge directe des nappes.L'évaporation cumulée pour les mois de Juin, Juillet et Août représente près de 50\% à l'échelle annuelle. Cette dernière calculée par la méthode Turc est de 1513,22mm pour la palmeraie de Touggourt, limite Sud de la vallée d'OuedRigh[13].

Du point de vue hydrologique l'axe de la vallée est occupé par le grand canal de drainage qui mène les eaux vers les chotts. Le flanc Ouest est parcouru par de petits oueds qui descendent de la dorsale mozabite, causés par des crues épisodiques.

Les Oueds El Attar, N'oura, Medjeiar, Mellah apportent, par leurs crues relativement fréquentes, des possibilités d'approvisionnement aux nappes de l'Oued Righ.

\subsection{Contexte hydrogéologique}

Le bassin versant de l'Oued Righ comblé de terrains Quaternaires (sables, calcaires, grès, argiles et évaporites) siège de la nappe superficielle (niveaux sableux et argilo-évaporitiques). L'alternance de couches imperméables et perméables d'une part et l'existence d'un fossé de subsidence d'autre part, ont favorisé la formation de deux grands systèmes aquifères captifs définis par le Complexe Terminal (CT) du Miopliocéne et le Continental Intercalaire (CI) formés entre les deux cycles marins du Paléozoïque et du Crétacé Supérieur constituent respectivement les limites inférieures et supérieures. Ces deux horizons se trouvent à des profondeurs relatives de $500 \mathrm{~m}$ et plus de $1500 \mathrm{~m}$ respectivement pour le CT et le CI.

\section{METHODOLOGIE}

Comme toute autre discipline scientifique basée sur la cartographie, l'hydrogéologie trouve dans la télédétection une source inépuisable de données spatialement localisée. Selon les objectifs assignés, de nombreux traitements peuvent être appliqués aussi bien sur les bandes brutes queles néocanaux.

Le processus d'interprétationdes images, qu'il soit visuel (composition colorée) ou automatique (classification) [14], transforme les données contenues dans l'image en éléments d'information rattachés à une localisation géographique [15].

Les données spatiales dont nous disposons sont des scènes multi-date acquises par LANDSAT5 TM et LANDSAT 7 ETM+ [16] qui couvrent l'ensemble du secteur sur une durée de 22 ans: la couverture la plus ancienne date du 07-01-1987, la couverture la plus récente date du 01-04-2009 et l'intermédiaire date de la fin des années 2000 (02-11-2000).

Ces images offrent une bonne résolution aussi bien spectrale que spatiale appropriées pour une bonne étude hydrogéologique [17]. 
Les capteurs TM (Thematic Mapper) et ETM+ (Enhanced Thematic Mapper Plus) de LANDSAT5 et LANDSAT7 respectivement fournissent des scènes multispectrales ( 6 bandes multispectrales: ETM 1, 2, 3, 4, 5, 7, à 30 m)et une bande thermique (ETM 6 à 120 m). Le capteur ETM+ de LANDSAT 7 fourni également une bande panchromatique (la bande ETM 8) à $15 \mathrm{~m}$ de résolution spatiale. Ce nombre important de canaux nous a permis d'effectuer de multiples combinaisons trichromiques (compositions colorées) mettant en évidence les différents thèmes [18]. Elles permettent d'obtenir la meilleure discrimination visuelle des objets de l'image, laissant à l'analyste le soin d'interpréter les variations des tons et des couleurs qui traduisent des variations de faciès ou des états de surface [19].

Dans le cadre de notre travail, nous avons retenu la combinaison des bandes ETM+ 5 ETM+ 4et ETM+3 affichées en rouge, vert et bleu respectivement, fortement conseillée par les ouvrages spécialisés de télédétection [20].

Cette trichromie permet également une bonne séparation des eaux temporaires vis à vis des autres systèmes d'occupation du sol, et une bonne discrimination visible entre les végétaux, les plansd'eauainsi que les surfaces bâties, principaux systèmes d'occupation du sol de notre zone d'étude(fig. 2).Ces états de surface ainsi discriminés ont servi pour l'étape décisive d'échantillonnage des classes, essentielle pour la classification supervisée.

La classification supervisée constitue la meilleure méthode d'extraction automatique des états de surface [21]ce qui la rend un procédé de choix pour répondre au besoin de leur cartographie automatique [14].

Elle consiste à regrouper des pixels en catégories les plus homogènes possibles, en créant des classes qui contiennent le plus de pixels similaires et représentant une même occupation du sol.

Plusieurs méthodes de classification ont été développées à savoir Mahalanobis, Maximum Likelihood, barycentique [22], Spectral Angle Mapper [23] et la classification par réseau neuronaux [24].

Dans le cadre de notre étude c'est la méthode dite du Support Vector Machine (SVM), initiées par Cortes \&Vapnick en 1995[25]qui a été la plus fiable. Les récentes recherches dont des études comparatives indiquent un fort potentiel de la classification par Machines à Vecteurs de Support (ou Séparateurs à Vastes Marges) et ont montré que cette méthode pouvait être plus probante que les techniques habituelles (comme la méthode des réseaux neuronaux ou les arbres de décision) et les classificateurs probabilistes traditionnels (tel que le maximum de vraisemblance) [14]. Elle fournit directement une cartographie définitive (fig. 3) en couleurs conventionnelles par pixel [26]. Des échantillons de terrains sont utilisés pour 
l'échantillonnage de vérification (des vérités terrain) nécessaire pour valider les résultats de ces classifications par les matrices de confusion.
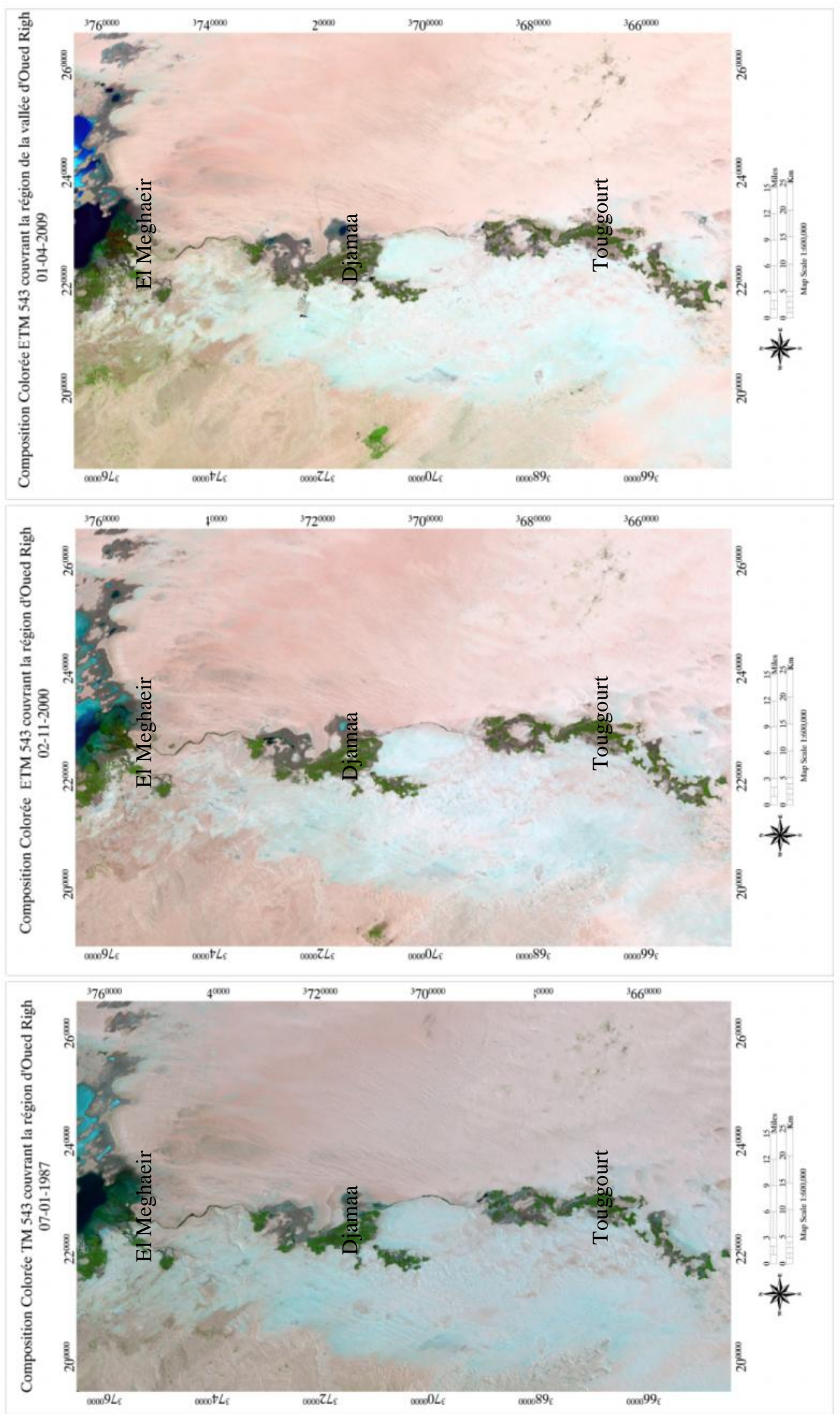

Fig.2.Compositions colorées543 de la région de la vallée d'Oued Righ en 1987-2000 et 2009. 


\section{RESULTATS ET DISCUSSION}

Les classifications obtenues pour l'ensemble des trois dates sont par la suite transférées vers un logiciel SIG pour obtenir des cartes d'occupation de sols, permettant d'identifier les diverses composantes de l'espace étudié (fig. 3).

Pour faciliter et synthétiser l'analyse de l'évolution globale de l'occupation du sol, dix classes ont été retenues: eau, eau salée saturé, sols humides, sels, sols salés secs, palmeraies, végétations, sables, grés rouges et agglomérations.

L'évaluation de nos résultats à partir des matrices de confusion, nous permet de constater que les précisions des classifications sont de $(97,04 \%$ pour la carte de $2009,96.44 \%$ pour la carte de 2000 et 95,71\% pour celle de 1987) et les indices Kappa sont respectivement 0.9654, 0.9565 et 0.9289 . les résultats indiquent que les classifications sont de bonne qualité avec un accord presque parfait entre le résultat et la vérité sur terrain [5].

L'étude du changement d'occupation du sol, repose sur l'utilisation de l'équation très courante proposée par la FAO en 1996 [27]. Les valeurs positives représentent une progression de la surface de la classe pendant la période étudiée et les valeurs négatives indiquent la perte de surface (régression). Les valeurs proches de zéro nous indiquent que la classe reste relativement stable entre les deux dates.

Considérant T1 l'ensemble de pixels a la date 1 (1987), T2 l'ensemble de pixels pour la date 2 (2000) et T3 l'ensemble de pixels pour la troisième date (2009) (Tableau. 1\&2), nous avons calculé les taux de changement entre les dates 1987 - 2000 (T2 - T1), les dates 2000 - 2009 (T3 - T2) et les dates 1987-2009 (T3 - T1) afin de montrer l'évolution de chaque classe au cours du temps. 


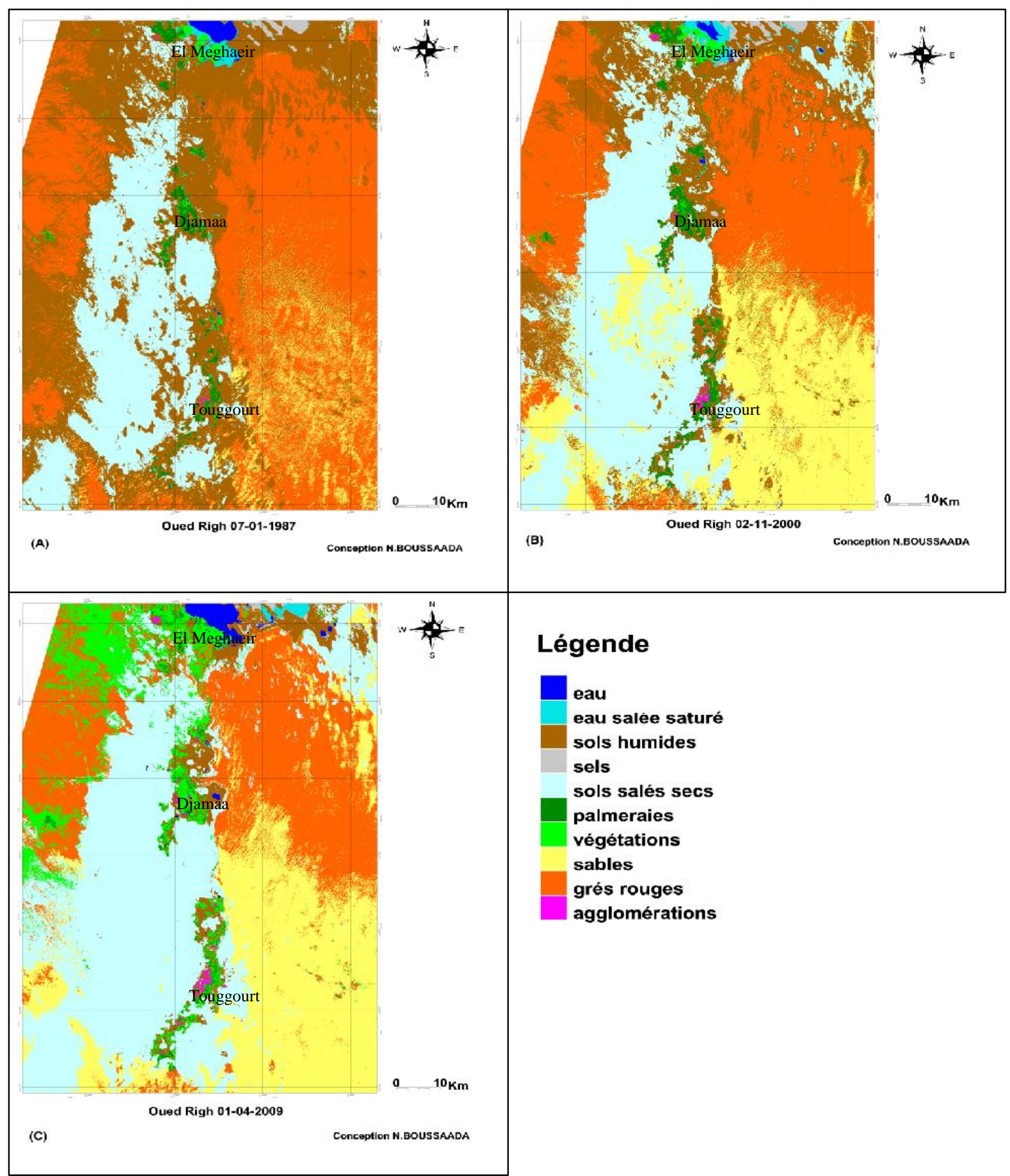

Fig.3. Cartes d'occupation du sol en 1987-2000 et 2009

Ces cartes montrent à la fois l'ampleur des pertes et gains en terres dans la zone étudiée ainsi que leur variabilité dans l'espace.

Elles permettent de connaître les tendances des changements de l'occupation du sol tout en considérant la répartition spatiale de ces changements. 
En effet, ces dernières tentent à mettre en évidence la relation entre l'homme et son milieu.

\subsection{Les changements spatio-temporels entre 1987 et 2000}

L'analyse diachronique souligne des évolutions contrastées selon les occupations du sol.

Les valeurs positives représentent une progression de la surface de la classe pendant la période analysée et les valeurs négatives indiquent la perte de surface d'une classe entre les deux dates. Les valeurs proches de zéro nous indiquent que la classe reste relativement stable entre les deux dates.

D’une situation dominante en 1987 (35\% de la superficie du site), les sols humides n'occupent en 2000 plus que $18 \%$, soit $1913 \mathrm{~km} 2$. Cette réduction de la superficie d'environ (47\%) serait imputable à la forte évaporation [13].

La classe de l'agglomération, initialement concentré à proximité des palmeraies, a subi de forts changements est s'est étendu dans toute la région. L'espace agricole s'est énormément accru lui aussi au cours de cette période. L'extension des palmeraies passe de $113 \mathrm{~km}^{2}$ en 1987 à $156 \mathrm{~km}^{2}$ en 2000soit un changement de $38 \%$ dans une période de 13 ans.

Cette évolution est essentiellement due à la forte croissance démographique d'une année à l'autre.

Par ailleurs, les sols à texture sableuse passant de $563 \mathrm{~km}^{2}$ à $2371 \mathrm{~km}^{2}$, mettent en valeur la mobilisation des éléments fins dans la région.

Autres classes qui montrent des forts changements sont la classe des eaux et la classe des sels. Une telle diminution des plans d'eau et l'accroissement du taux des sels à la surface serait due à la date de prise de vue (Janvier 1987 -Novembre 2000), la faiblesse des précipitations et le taux d'évaporation intense. Cette explication nous parait judicieuse, dans la mesure que la zone d'étude est caractérisée par un climat hyper aride.

Les taux de changement entre les surfaces des classes d'occupation du sol entre les deux dates sont indiqués dans le tableau ci-dessous. 
Tableau 1. Changement d'occupation du sol entre 1987 et $2000 . \mathrm{T} 1=$ surface à la date $1 ; \mathrm{T} 2$ $=$ surface à la date $2 ;$ Tc1 = taux de changement entre les dates 1987 et 2000.

\begin{tabular}{llclccc}
\hline \multirow{2}{*}{ classes } & $\mathbf{1 9 8 7}$ & \multicolumn{3}{c}{$\mathbf{2 0 0 0}$} & & \\
\cline { 2 - 5 } & $\begin{array}{l}\text { Surface } \\
\text { en km }\end{array}$ & pourcentage & $\begin{array}{l}\text { Surface } \\
\text { en km }\end{array}$ & Pourcentage & T2-T1 & Tc1 \\
\hline Eau & & & & & & \\
Eau salée saturé & 44.75 & 0.44 & 17.84 & 0.18 & -26.91 & -0.60 \\
& 47.01 & 0.47 & 45.84 & 0.45 & -1.17 & -0.025 \\
Sols humides & 3577.61 & 35.39 & 1913.77 & 18.93 & -1663.84 & -0.47 \\
Sels & 42.05 & 0.42 & 67.84 & 0.67 & 25.79 & 0.61 \\
Sols salés secs & 2069.10 & 20.47 & 2795.80 & 27.66 & 726.7 & 0.35 \\
Palmeraies & 113.34 & 1.12 & 156.67 & 1.55 & 43.33 & 0.38 \\
Végétations & 55.26 & 0.55 & 73.65 & 0.73 & 18.39 & 0.33 \\
Sables & 563.13 & 5.57 & 2371.68 & 23.46 & 1808.55 & 3.21 \\
Grés rouges & 3592.94 & 35.54 & 2653.92 & 26.25 & -939.02 & -0.26 \\
Aggglomération & 3.58 & 0.03 & 11.76 & 0.12 & 8.18 & 2.28 \\
Total & 10108.77 & 100 & 10108.77 & 100 & & \\
\hline
\end{tabular}

\subsection{Les changements spatio-temporels entre 2000 et 2009}

Le tableau 2 révèle un accroissement des surfaces des agglomérations au bout de 09 ans (soit une progression de $67 \%$ ).

La croissance de la population engendre d'importants problèmes environnementaux (rejet d'eaux usées directement dans nature, pollution, salinisation des sols....etc)

Le site est caractérisé par un déclin important des palmeraies $\left(62 \mathrm{~km}^{2}\right.$ en 2009 contre $156 \mathrm{~km}^{2}$ en 2000, soit une diminution de $60 \%$ ).

Une hausse importante de la classe des eaux (soit plus de $64 \mathrm{~km}^{2}$ ) est expliquée par les rejets domestiques et des eaux de drainage dans la nature, par conséquent la mort du palmier dattier par asphyxie (diminution de la classe des palmeraies). Dans certaines zones, il y a un nombre élevé de forages, parfois à moins de 15 mètres l'un de l'autre. La nappe est alors rabattue d'une façon remarquable.

La progression des sols salés dans la région, nettement apparente, est explicable par son climat hyper aride (la très forte évaporation), la qualité chimique des eaux des nappes, notamment celle du complexe terminal (relativement chargée) ainsi que la nature des terrains et surtout la concentration des sels dans le temps.

Le problème de salinité des sols s'est aggravé suite à l'utilisation des eaux salées pour l'irrigation avec un drainage défectueux. 
Tableau 2. Changement d'occupation du sol entre 2000 et 2009. T2 = surface à la date 2 ; T3 $=$ surface à la date $3 ;$ Tc2 = taux de changement entre les dates 2000 et 2009.

\begin{tabular}{|c|c|c|c|c|c|c|}
\hline \multirow[b]{2}{*}{ classes } & \multicolumn{2}{|c|}{2000} & \multicolumn{2}{|c|}{2009} & \multirow[b]{2}{*}{ T3-T2 } & \multirow[b]{2}{*}{ Tc2 } \\
\hline & $\begin{array}{l}\text { Surface en } \\
\mathbf{k m}^{2}\end{array}$ & pourcentage & $\begin{array}{l}\text { Surface } \\
\text { en } \mathbf{k m}^{2}\end{array}$ & pourcentage & & \\
\hline Eau & 17.84 & 0.18 & 82.62 & 0.82 & 64.78 & 3.63 \\
\hline eau salée saturé & 45.84 & 0.45 & 25.63 & 0.25 & -20.21 & -0.44 \\
\hline sols humides & 1913.77 & 18.93 & 454.89 & 4.50 & -1458.88 & -0.76 \\
\hline sels & 67.84 & 0.67 & 29.41 & 0.30 & -38.43 & -0.57 \\
\hline sols salés secs & 2795.80 & 27.66 & 3565.20 & 35.27 & 769.4 & 0.28 \\
\hline palmeraies & 156.67 & 1.55 & 62.82 & 0.62 & -93.85 & -0.6 \\
\hline végétations & 73.65 & 0.73 & 777.94 & 7.69 & 704.29 & 9.56 \\
\hline sables & 2371.68 & 23.46 & 2506.62 & 24.80 & 134.94 & 0.06 \\
\hline grés rouges & 2653.92 & 26.25 & 2584.02 & 25.56 & -69.9 & -0.03 \\
\hline agglomération & 11.76 & 0.12 & 19.62 & 0.19 & 7.86 & 0.67 \\
\hline Total & 10108.77 & 100 & 10108.77 & 100 & & \\
\hline
\end{tabular}

\subsection{Bilan final de l'évolution de l'occupation du sol}

La surface de l'eau présente des d'importantes fluctuations dont l'augmentation pour l'année 2009. En 2009(82.62 km²), il y a à peu près deux fois plus d'eau qu'en 1987(44.75 km²), soit un gain de $37.87 \mathrm{~km}^{2}$ (fig.4). Une diminution de $3122.72 \mathrm{~km}^{2}$ du territoire occupé par les sols humides est par ailleurs notée.

Ces variations pourraient être attribuables soit à l'intensité de l'évaporation (hausse des températures) soit à la transformation de ces sols en autre classe. L'une des conséquences visible de cette situation est observable au niveau de l'apparition de nouveaux plans d'eau à la surface(remontée des eaux). Au départ, l'eau couvrait une superficie estimée à $44.75 \mathrm{~km}^{2}$ pour une superficie de $82.62 \mathrm{~km}^{2}$ en 2009.

Le nombre de la population est marqué par une augmentation spectaculaire entre 1987 et 2005 qui, de 203087 personnes en 1987et 280824 personnes en1998 [28], dépassent les 356479 personnes en 2005 [29].Ces importants changements dans le nombre des populations (agglomérations) s'expliquent en réalité par le développement des infrastructures.

Entre 2000 et 2009, la végétation représentée par un maquis, augmente nettement (fig. 4). L'augmentation de ce milieu peut être liée à sa confusion avec la classe eau. La difficulté à discriminer visuellement ces deux classes peut être due à leurs signatures spectrales comparables. Cette confusion peut s'expliquer également par leur présence conjointe due au fait que ces végétaux poussent en présence de grandes quantités d'eau. L'activité humaine 
(rejet des eaux directement dans la nature) contribue donc à augmenter notablement la densité du maquis.
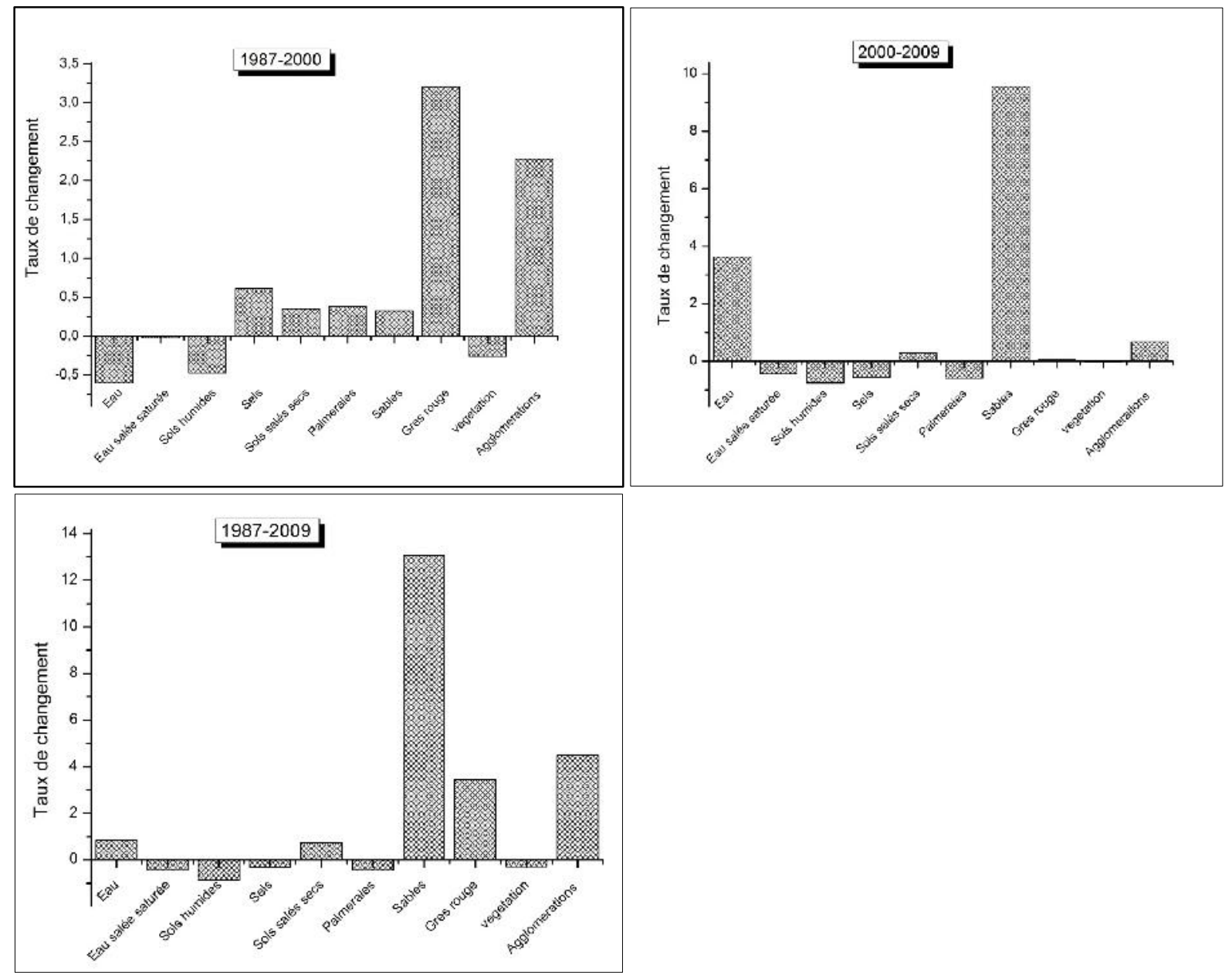

Fig.4. Bilan final du taux de changement d'occupation de sol (1987-2000 et 2009).

\section{CONCLUSION}

La présente étude confirme le potentiel des images satellites qui s'avèrent très efficaces pour l'appréhension des transformations globales de l'occupation du sol et leurs conséquences [30], présentant ainsi une source de données qui devient de plus en plus indispensable en hydrogéologie [31-32].

Associée aux études de terrains, l'imagerie satellitaire assure la surveillance régulière de l'environnement permettant une compréhension, à la fois fine et globale, des modifications profondes de l'occupation du sol. Ensemble, ils constituent un enjeu important pour l'évaluation du degré de vulnérabilité de l'environnement face à la pollution et la salinité des sols. 
L'analyse diachronique d'images satellitaires, réalisée en vue d'évaluer la dynamique de l'occupation du sol et son impact sur l'environnement, montre des changements importants dans l'occupation du sol du site étudié. En effet, la croissance démographique engendre une élévation de l'utilisation des eaux et par conséquent d'importantes quantités d'eaux excédentaires issues de l'irrigation des palmeraies et des eaux usées se déversent dans la nature. Celles-ci augmentent ainsi la salinité des sols et provoquent l'asphyxie des palmeraies. On peut conclure que les modifications de l'occupation du sol dans la zone d'étude sont assez nettes et se traduisent de façon conséquente par les changements dans l'espace géographique et l'évolution des superficies.

Pour améliorer nos résultats, nous recommandons, l'utilisation d'images à très haute résolution spatiale (de l'ordre du mètre) [33-36] qui offriront une discrimination optimale des états de surface et donc un meilleur suivi.

\section{REMERCIEMENT}

Ce travail est le fruit des travaux de stages effectués au sein du Centre des études Spatiales de la Biosphère (CESBIO). Les auteurs tiennent à remercier et présentent leur profonde gratitude à Mme Danielle Ducrot pour son aide, son encouragement et ses conseils.

Nos remerciements infinis et respect les plus sincères vont directement à Mr Yann Kerr pour son accord d'accueil.

\section{REFERENCES}

[1] Lili -Chabaane Z, Bergaoui H, Ouirghi H. Télédétection et analyse spatiale pour l'évaluation des besoins en eau agricole à l'échelle régionale : cas de la plaine de Kairouan dans le centre de la Tunisie. Les XIèmes Journées Scientifiques du Réseau Télédétection de l'AUF ; Les milieux naturels et agricoles, 2008, 128-129.

[2] El Hadani D.Télédétection et système d'information géographique pour la gestion et la recherche de l'eau. (Proceedings of Rabat Symposium S3,). IAHS Publ., 1997, no. 242,197204.

[3] Andrieu J, Mering C. Cartographie par télédétection des changements de la couverture végétale sur la bande littorale ouest-africaine : exemple des rivières du sud du delta du Saloum (Sénégal) au rio Gêba (Guinée-Bissau). Revue Télédétection., 2008, vol. 8, n 2 ,93118.

[4] Benhadj I, Duchemin B, Maisongrande Ph, Simonneaux V, Mougenot B, Khabba S, Chehbouni G.Dé convolution d'image multi temporelle MODIS pour la détermination de 
l'occupation du sol et du bilan hydrique à l'échelle régionale (plaine du Tensift, Maroc central). XIèmes Journées Scientifiques du Réseau Télédétection de l'AUF Télédétection et gestion de l'environnement, 2008, 12-13.

[5] Chalifoux S, Nastev M, Lamontagne C, Latifovic R, Fernandes R. Cartographie de l'occupation et de l'utilisation du sol par imagerie satellitaire LANDSAT en hydrogéologie. Télédétection, 2006, Vol. 6, n 1, 9-17.

[6] Corgne S, Magagi R,Sylla D,Yergeau M. Fusion de données multi source pour la détection de linéaments hydrogéologiques: Application en milieu semi-aride (Niger). XIèmes Journées Scientifiques du Réseau Télédétection de l'AUF, Télédétection et gestion de l'environnement, 2008), 18-20.

[7] Hoang K.H, Bernier M, Villeneuve J.P. Les changements de l'occupation du sol et ses impacts sur les eaux de surface du bassin versant de la rivière Câu (Viêt-nam). Mémoires de maitrise, 2007, pp.127

[8] Soro G, Ahoussi E.K, Kouadio E.K, Soro T.D, Oulare S, Saley M.B, Soro N, Biemi J. Apport de la télédétection à la cartographie de l'évolution spatio-temporelle de la dynamique de l'occupation du sol dans la région des Lacs (Centre de la Côte d'Ivoire). Afrique SCIENCE10 (3) ,2014, 146-160.

[9] Zombre P. N. Evolution de l'occupation des terres et localisation des sols nus dans le centre nord du Burkina Faso. Télédétection, 2006, vol. 5, n4 , 285-297.

[10] Zribi M. Fonctionnement et ressources hydro-écologiques en région semi-aride (Tensift, Maroc) Caractérisation, modélisation et prévision. Sud-Med Bilan du projet 2 CESBIO, $2010,119-152$.

[11] Observatoire du Sahara et du Sahel (OSS). Amélioration de la connaissance et de la gestion concertée du système Aquifère du Sahara Septentrional (SASS) par l'utilisation des images satellitaires : étude de cas, GEOAQUIFER, Cartographie des usages de l'eau par télédétection dans un bassin transfrontière: le Système Aquifère du Sahara Septentrional. Facilité africaine de l'eau, (Eds.) 2010, pp. 22

[12] Chenchouni H. Diversité floristique d'un lac du bas-Sahara algérien. Acta Botanica Malacitana, Málaga 37, 2012, 33- 44.

[13] Dubost G, Dubost D. Méthodes pratiques pour le calcul de l'évapotranspiration au Sahara algérien. Bul. Agro. Sah, N5, 1986, 36-52.

[14] Hammad N. Cartographie géologique et analyse linéamentaire de la région d'El Kseïbat (Sahara du sud-ouest) à partir des images spatiales. Incidence sur l'exploration minière. Thèse Doctorat es Science, Université Ouargla, Algérie, 2016, pp.188. 
[15] Noyola-Medrano C, Mering C, Rojas-Beltrán M.A.Evaluation du changement de l'occupation du sol à l'aide des images LANDSAT et SPOT : champ volcanique de la sierra chichinautzin (MEXIQUE). Actes du 26eme colloque international; cartographique conférence, Allemagne du 25 au 30 Aout 2013 ,1-12.

[16] Bannari A, Teillet Ph.M, Landry R. Comparaison des réflectances de surfaces naturelles dans les bandes spectrales homologues des capteurs TM de 1ANDSAT-5 et ETM+ DE LANDSAT-7. Télédétection, vol. 4, n³, 2004, 263-275.

[17] Youan-Ta M, Lasm T, Jourda J.P, Kouame K.F, Razack M. Cartographie des accidents géologiques par imagerie satellitaire landsat-7 ETM+ et analyse des réseaux de fractures du socle précambrien de la région de Bondoukou (Nord-est de la côte d'ivoire). Revue Télédétection, vol. 8, $\mathrm{n}^{\circ}$ 2, $2008,119-135$.

[18] Sarr M.A. Cartographie des changements de l'occupation du sol entre 1990 et 2002 dans le nord du Sénégal (Ferlo) à partir des images Landsat. Article 472 Paru dans Cybergeo : European Journal of Geography , Environnement, Nature, Paysage,2009, pp.16

[19] Donnay J.P. Les spatiocartes en composition colorée. Bulletin de la Société Géographique de Liège, 38, 2000/1, 43-61.

[20] Girard M-C, Girard, C-M. La télédétection appliquée, zones tropicales et intertropicales, Paris, (Eds.) 1999, pp. 529.

[21] Ducrot D. Méthodes d'analyse et d'interprétation d'images de télédétection multi-sources. Extraction de caractéristiques de paysage. Mémoire d'habilitation à diriger des recherches, INP Toulouse, 2005, pp. 210.

[22] Richards J.F. "Land transformation". The earth as transformed by human action. Ed. by Turner B.L. II, Clarck W.C, Kates R.W, Richards J.F, Mathews J.T, Meyer W. Cambridge University Press, Cambridge, 1990, 163-178.

[23] Kruse F. A, Lefkoff A, B. Boardman J. B, Heidebrecht K. B, Shapiro A. T, Barloon P. J, and Goetz A. F. H. The Spectral Image Processing System (SIPS) Interactive visualization and Analysis of Imaging Spectrometer Data. Remote Sensing of the Environment, v. 44, 1993, 145-163.

[24] Lippmann R. An introduction to computing with neural nets, IEEE Assp magazine, vol. 4, issue 2, 1987, 9- 22 .

[25] Cortes C, Vapnik V. Support-Vector networks. Machine Learning, 20 (3), 1995, 273297. 
[26] Mequignon L, Croisille G, Lejeune V. Application de la télédétection à l'étude des zones humides : identification des prairies, des roselières, des peupleraies et des gravières. Document technique, 2005, pp.134.

[27] FAO Forest resources assessment 1990. Survey of tropical forest cover and study of change processes. Forestry Paper, 130, Roma, 1990, pp. 152.

[28] Kouzmine Y. Dynamiques et mutations spatiales du Sahara algérien, vers de nouvelles approches fondées sur l'observation, thèse de doctorat en géographie, 2007. Laboratoire ThéMA, Université de Franche-Comté, pp. 423.

[29] D.P.A.T. Annuaire statistique 2005 de la wilaya d'Ouargla. Direction de la planification et de l'aménagement du territoire de la wilaya d'Ouargla, 2006, pp.192.

[30] Benmessaoud H, Kalla M, Driddi H. Evolution de l'occupation des sols et désertification dans le Sud des Aurès (Algérie). M@ppemonde94, 2009, 1-11.

[31] King C.États de surface et ruissellement, l'approche par télédétection. La Houille Blanche, Revue Internationale de l'Eau, n¹, 2002, 41-48.

[32] Olioso A, Jacob F.Estimation de l'évapotranspiration à partir de mesure de télédétection. La Houille Blanche, Revue Internationale de l'Eau, n¹, 2002, 62-67.

[33] Franklin S.E, Connery D.R, Williams J.A. Classification of alpine vegetation using Landsat Thematic Mapper, SPOT HRV and DEM data. Canadian Journal of Remote Sensing, 20, 1994, 49- 56.

[34] Desclee B, Defourny P, Bogaert P. Identifying Forest Dynamics for Change Detection Using Multitemporal SPOT Imagery. IEEE International Workshop on the Analysis of Multitemporal Remote Sensing Images, 2007, 1- 6.

[35] Fengping D, Xiaowen L, Chongguang Z. A New Method in Change Detection of Remote Sensing Image. CISP'09, IEEE 2nd International Congress on Image and Signal Processing, 2009, $1-4$.

[36] RakotomalalaF.A. Evaluation de l'apport d'images à Très Haute Résolution Spatiale pour la modélisation de l'occupation du sol : cas du complexe d'Aires Protégées de Zahamena. Mémoire de DEA (Diplôme d'Etudes Approfondies) en Géophysique, Faculté des Sciences, Université d'Antananarivo, 2012, pp. 80. 


\section{How to cite this article:}

Boussaada-maabdi N, Bousnoubra-Kherici H, Kherici N, Hammad N. Mapping of land-cover from remotely sensed images in the valley of oued righ (algerian southeast). J. Fundam. Appl. Sci., 2017, 9(2), 696-712. 